\title{
Optimizing Allocation of Course Resources in the Colleges of Science and Engineering: a Case Study on the Course Enrollment Database of a University in Beijing
}

\author{
Xiaoqi Che ${ }^{1 *}$, Yang $\mathrm{Xiao}^{2}$, Long $\mathrm{Su}^{1}$ and Lei $\mathrm{Bao}^{3}$ \\ ${ }^{1}$ Beihang University, Beijing 100191, China \\ 2 South China Normal University, Guangzhou 510631, China \\ 3 The Ohio State University, Columbus, Ohio 43210, USA \\ *Correspondence: chexiaoqi520@126.com
}

(Received: 01/15/2020; Accepted: 04/21/2020; Published: 04/28/2020)

DOI: https://doi.org/10.37906/real.2020.4

\begin{abstract}
A course selection (CS) model is constructed based on the course enrollment database from a Chinese university in this research. By using big-data analysis on the database, we study the factors that may determine the students' CS and we investigate how to optimize allocation of course resources. Our results reveal that the allocation of course resources (type of courses) and the recent revision of academic programs will significantly influence students' CS. Courses on natural science and engineering (type-b courses), as well as on literature and art (type- $\mathrm{d}$ courses), are becoming most popular in course enrollment, which are followed by practical employment-related courses (type-c courses) such as law and management. The core courses of the major (type-a courses) are least selected. Since the new academic program was implemented, type-b courses have been becoming more popular but type-c and type-d courses are less enrolled than before. Our findings can be referenced by policy makers to improve the allocation of course resources in universities, which will benefit the students' CS and satisfy the demands from society. Our results will also serve as important information for improving education efficiency.
\end{abstract}

Keywords: allocation of Course Resources; educational efficiency; course selection; big data analysis; credit system

\section{Introduction}

The top talent training has been one of the key criteria to evaluate the ranking of a university in the world. The fundamental task of talent training is to serve the key national strategies and the needs of economic and social development. As the core element of talent training, curriculum addresses the most fundamental issues. Therefore, great emphasis has been placed on "curriculum construction", which deals with not only the curriculum quality, but also the curriculum education efficiency mainly affected by curriculum resource allocation. According to the principles of talents training and discipline development trends, it is important to optimize curriculum resource allocation to meet the needs of economic and social development, and endow students with more "learning autonomy" through implementation of credit system. The relationship is shown in Figure 1.

As for curriculum resource allocation, colleges and universities can thus carry out the overall design for the course offering based on their understanding of national and social needs. Besides, students' 
course selection (CS) behaviors may reflect the needs of economic and social development to a certain extent, and the greater the degree of CS autonomy, the more real the needs of economic and social development can be reflected. Therefore, colleges and universities can also optimize the allocation of course resources according to students' CS behaviors.

Even though a number of studies have been devoted to the top-down theoretical models based on national strategies and university construction goals, bottom-up studies reflecting the allocation of curriculum resources from perspectives of school policies and students' CS behaviors are rare. As a result, this study analyzes the CS data of four departments in the college of science and engineering from a university in Beijing, and explore the influence of course resource allocation and social needs on students' CS behaviors under the background of credit system implementation. This study may provide reference for course resource allocation policies to achieve the matching of curriculum resource allocation with students' CS needs and social development needs, promoting curriculum education efficiency and the quality of curriculum construction.

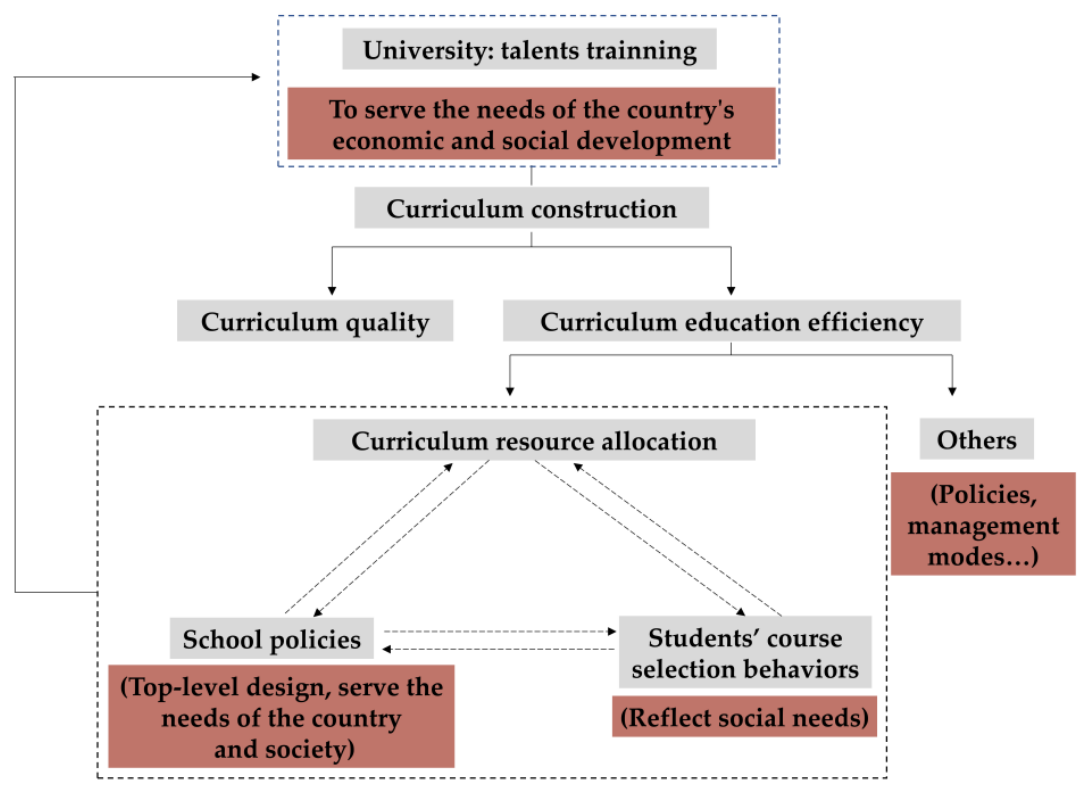

Figure 1. Curriculum resource allocation model

\section{Backgrounds}

As a complex decision-making process affected by multiple factors, course selection (CS) is a process in which students weigh various types of CS information (Babad \& Tayeb, 2003; Pass, Mehta \& Mehta, 2012). In the past research, Babad et al. (2003) designed virtual courses for students to choose in a simulation. Based on the analysis of students' CS, they summarized the attributes of students' selected courses and corresponding influencing factors. It shows that the factors influencing college students' curriculum choice can be divided into academic considerations - such as curriculum content, difficulty and teaching mode - and personal/situational considerations - such as students' desire for high-paying jobs and social development needs. In addition, a multitude of research efforts have been directed by means of questionnaire to investigate students' motivation of CS and to explore the influencing factors. Roger Kerin et al. (1975) pointed out that students mainly consider factors such as personal interest, course content, compatibility with their majors and teacher in CS. Moreover, Babad et al. (1999) identified 
that students' curriculum choice would be affected by a myriad of factors such as teacher, curriculum and individual, among which interest, desire to learn, teachers' attitude, passing rate of examination are the most concerned ones. However, the research by McGoldrick et al. (2002) and Babad (2001) reveals that practical factors such as professional development, employment and social demand would greatly influence students' curriculum choice.

It should be noted that the above two research methods are limited by the rationality and effectiveness of virtual curriculum or questionnaire design, as well as cooperation of the subjects (Kelle, 2006). Although the simulated CS based on virtual courses has restored the CS process, it ignores possible factors in real life. Also, though the questionnaire survey may record students' CS influencing factors as much as possible, it lacks the physical experience of CS in real life, thus fails to fully reflect students' CS process.

\section{Research design}

3.1 Curriculum resource allocation model and the influencing factors of students' CS

\subsubsection{Curriculum resource allocation model}

As mentioned above, curriculum construction is composed of curriculum quality construction and curriculum education efficiency. Affected by policy, management mode and resource allocation, etc., the efficiency of curriculum education is mostly influenced by the allocation of curriculum resources, which includes curriculum content design and allocation of teaching resources, etc. From the perspective of economics and management, curriculum resource allocation, school policy, and students' CS behaviors interact with one another. The optimization of curriculum resource allocation results from school policy and students' CS behavior. Therefore, the influence of school policy on curriculum resource allocation is similar to the planning allocation mechanism, while the influence of students' CS behavior is comparable to the market mechanism (Calvo \& Frenkel, 1991; Marginson, 2013). In other words, the allocation of curriculum resources depends on both school policies and students' CS behaviors (i.e. what kind of courses and styles students are willing to choose will in turn affect which courses schools prefer to offer). Students' actual selection of courses is the result of the balance between policy influence and curriculum resource allocation, which to a certain extent reflects the needs of social development. Meanwhile, the current situation of resource allocation and students' CS may serve as an indicator of how reasonable school policies are, and be used as a guide to future policy revision. The relationship is shown in Figure 1.

\subsubsection{The influencing factors of students' CS}

As there are many factors (variables) influencing students' CS, the factors (variables) were simplified and divided into two categories when constructing the CS model: school's resource allocation and external factors. The resource allocation of schools involves the number and type (content) of courses that schools can provide, while the external factors mainly include personal interests, social considerations such as economic and social development needs, employment needs and personal development, and national and school policies, etc.

\subsection{Research method}

Different from the previous studies focusing on virtual CS, we explore the factors that may affect students' CS in real life by analyzing the real data of students' CS in a university. Based on the CS model described above, we assume that students' CS behaviors may be affected by resource allocation and external factors, each of which covers many sub-factors. In addition, as exhibited in previous studies, CS is affected by multiple factors simultaneously, such as course type, and personal and social situations. To simplify the variables, this study takes curriculum type as major resource allocation influencing factor, 
and academic program as the main external factor. For sampling comprehensiveness, personal interest and other factors are regarded as random errors. In this study, the quantitative evaluation index of students' preference for curriculum is CS rate.

\subsubsection{Research object and data source}

The object of this study is undergraduates $(N=2548)$ enrolling from 2013 to 2016 in four departments (Department of Materials, Department of Aeronautics, Department of Instrumentation and Department of Physics) of a university in Beijing. The four departments are all of interdisciplinary characteristics, and students enjoy greater CS autonomy. The CS data comes from students' selection results from Autumn 2013 to Spring 2018 regarding general education courses, public elective courses and interdisciplinary courses (professional courses of other majors). In 2015, the university has substantially adjusted its academic program, and thus the 2013 and 2014 undergraduates were divided into the first group using the old version of academic program $\left(\mathrm{N}_{1}=1345\right)$; and the 2015 and 2016 undergraduates constituted the second group using the new version of academic program $\left(\mathrm{N}_{2}=1203\right)$. Compared with the old academic program, the new one is completely based on credit system, dividing courses into basic, general and professional ones. At the same time, CS autonomy is emphasized, allowing students to take professional courses in other departments.

\subsubsection{Course type and CS rate}

According to the International Standard Classification of Education (ISCED) issued by UNESCO, the types of talent training in higher education are divided into talents for academic research, talents for knowledge application and talents with vocational skill (ISCED, 1997). At present, talents for academic research and knowledge application are two main types of talent training in colleges and universities from China, of which cultivation of academic talents is relatively simple (mainly focusing on in-depth study of professional knowledge). However, application talents should possess comprehensive abilities such as business ability, communication ability, management ability and legal awareness, apart from mastery of professional and high-tech knowledge. Therefore, according to the correlation between curriculum and talent training, this study divided all courses into four categories: (a) the courses directly related to the major, (b) the courses strongly related to the major (STEM curriculum), (c) the courses weakly related to the major (employment-related courses such as law, economy and language), and (d) other courses (such as literature and art). Type-a courses are supplements of professional courses, most of which are offered by professional teachers to cultivate academic research talents. Type-b and type-c courses, respectively, refer to STEM courses such as natural science and engineering technology courses, and application courses such as literature and management courses, cultivating knowledge application talents. Type-d courses are general courses. This is also in good agreement with the factors which would influence students' decision-making of career life, such as interests, self-efficacy, values, etc. (Lent, Brown \& Hackett, 2000; Albion \& Fogarty, 2002).

On this basis, this study defined CS rate $S j(j=a, b, c, d)$ as "the percentage of a certain type of course selected by specific students in the total number of four types of courses".

\section{Analysis and Results}

To explore the relationship between students' CS behaviors with curriculum resource allocation and external factors, this study mainly investigates the differences of the CS rate concerning the four types of courses before and after the revision of academic program. The data analysis was carried out in SPSS24.0.

4.1 The interaction between course type and academic program revision on CS rate 
Two-way Repeated Measures ANOVAs were executed for the four departments, respectively, to test the interaction between the two factors which are course type and the revision of academic program. It is found that the interaction is statistically significant on the results for all these four schools, but the magnitude of influence differs. For Department of Physics, these two factors can explain $5.1 \%$ of the outcome variable variance $(p<0.001)$, which is close to the medium effect size. For Department of Materials and Department of Aeronautics, these two factors explain 11.8\% and $13.5 \%$ of the CS rate variance, respectively, which are close to the large effect size $(p<0.001)$. For Department of Instrumentation, the explanation effect of these two factors counts the strongest (20.6\%), which constitutes the large effect size $(p<0.001)$. Figure 2 intuitively presents this variation.
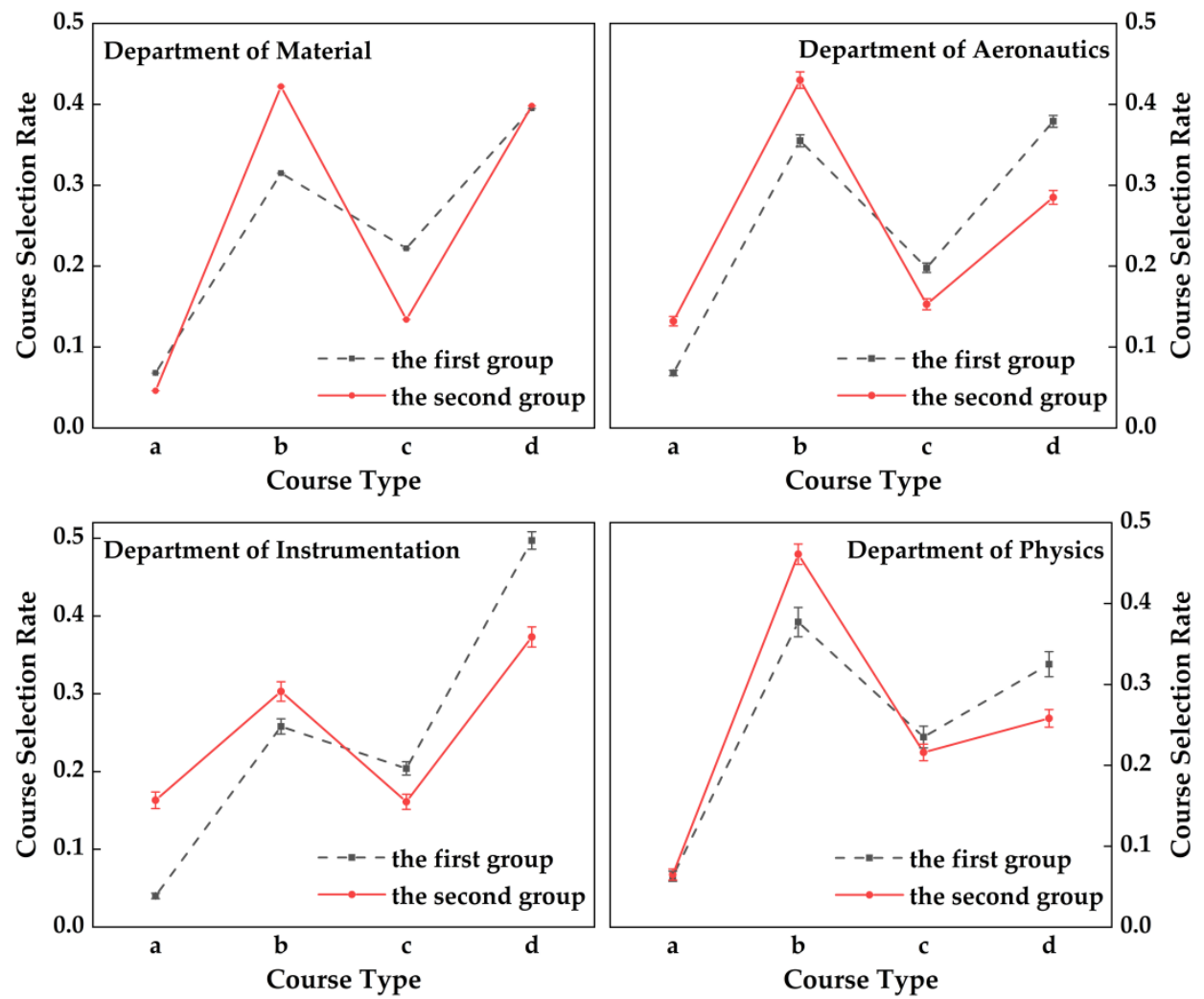

Figure 2. Interaction of course types and academic programs on the selection rate of the four types of courses in each department

Table 1 presents the selection rate of different types of courses for students of each department before and after the academic program revision. In general, after the revision of the academic program, the selection rate of type-b course (practical courses strongly related to their majors) of all the four department students has increased. 
Table 1. Mean and Standard Deviation (SD) of the selection rate (Sj) of the four types of courses before and after the academic program revision

\begin{tabular}{|c|c|c|c|c|c|c|c|}
\hline \multirow{2}{*}{ Department } & \multirow{2}{*}{$\begin{array}{c}\text { CS } \\
\text { rate* }\end{array}$} & \multicolumn{3}{|c|}{ The first group } & \multicolumn{3}{|c|}{ The second group } \\
\hline & & $\mathbf{n}$ & Mean & SD & $\mathbf{n}$ & Mean & SD \\
\hline \multirow{5}{*}{ Materials } & $\mathrm{Sa} \downarrow$ & \multirow{4}{*}{304} & 0.068 & 0.004 & \multirow{4}{*}{298} & 0.046 & 0.006 \\
\hline & $\mathrm{Sb} \uparrow$ & & 0.315 & 0.009 & & 0.422 & 0.012 \\
\hline & $\mathrm{Sc} \downarrow$ & & 0.222 & 0.007 & & 0.134 & 0.009 \\
\hline & $\mathrm{Sd} \rightarrow$ & & 0.395 & 0.009 & & 0.398 & 0.011 \\
\hline & & \multicolumn{3}{|c|}{$\mathrm{Sd}>\mathrm{Sb}>\mathrm{Sc}>\mathrm{Sa}$} & \multicolumn{3}{|c|}{$\mathrm{Sb} \approx \mathrm{Sd}>\mathrm{Sc}>\mathrm{Sa}$} \\
\hline \multirow{5}{*}{ Aeronautics } & $\mathrm{Sa} \uparrow$ & \multirow{4}{*}{559} & 0.068 & 0.077 & \multirow{4}{*}{466} & 0.132 & 0.125 \\
\hline & $\mathrm{Sb} \uparrow$ & & 0.355 & 0.176 & & 0.43 & 0.22 \\
\hline & $\mathrm{Sc} \downarrow$ & & 0.198 & 0.14 & & 0.153 & 0.147 \\
\hline & $\mathrm{Sd} \downarrow$ & & 0.379 & 0.173 & & 0.285 & 0.185 \\
\hline & & \multicolumn{3}{|c|}{$\mathrm{Sd} \approx \mathrm{Sb}>\mathrm{Sc}>\mathrm{Sa}$} & \multicolumn{3}{|c|}{$\mathrm{Sb}>\mathrm{Sd}>\mathrm{Sc} \approx \mathrm{Sa}$} \\
\hline \multirow{5}{*}{$\begin{array}{c}\text { Instrumenta } \\
\text { tions }\end{array}$} & $\mathrm{Sa} \uparrow$ & \multirow{4}{*}{339} & 0.04 & 0.067 & \multirow{4}{*}{287} & 0.163 & 0.182 \\
\hline & $\mathrm{Sb} \uparrow$ & & 0.258 & 0.181 & & 0.303 & 0.212 \\
\hline & $\mathrm{Sc} \downarrow$ & & 0.204 & 0.158 & & 0.161 & 0.165 \\
\hline & $\mathrm{Sd} \downarrow$ & & 0.497 & 0.206 & & 0.373 & 0.218 \\
\hline & & \multicolumn{3}{|c|}{$\mathrm{Sd}>\mathrm{Sb}>\mathrm{Sc}>\mathrm{Sa}$} & \multicolumn{3}{|c|}{$\mathrm{Sd}>\mathrm{Sb}>\mathrm{Sc} \approx \mathrm{Sa}$} \\
\hline \multirow{5}{*}{ Physics } & $\mathrm{Sa} \rightarrow$ & \multirow{5}{*}{143} & 0.063 & 0.074 & \multirow{5}{*}{152} & 0.065 & 0.085 \\
\hline & $\mathrm{Sb} \uparrow$ & & 0.377 & 0.217 & & 0.461 & 0.155 \\
\hline & $\mathrm{Sc} \rightarrow$ & & 0.235 & 0.16 & & 0.216 & 0.127 \\
\hline & $\mathrm{Sd} \downarrow$ & & 0.325 & 0.185 & & 0.258 & 0.135 \\
\hline & & & \multicolumn{2}{|c|}{$\mathrm{Sb} \approx \mathrm{Sd}>\mathrm{Sc}>\mathrm{Sa}$} & & \multicolumn{2}{|c|}{$\mathrm{Sb}>\mathrm{Sd} \approx \mathrm{Sc}>\mathrm{Sa}$} \\
\hline
\end{tabular}

*Note: “ $\uparrow, \downarrow, \rightarrow$ " respectively indicate that after the academic program was revised, the CS rate has significantly increased, decreased, and almost unchanged.

4.2 The effect of academic program revision on CS rate

Independent $\mathrm{t}$-tests were conducted to test for academic program differences in CS rate of four types, respectively. Among the four types of courses, the CS rate of the practical courses that are strongly related to the major (type b) consistently exhibited a significant increase in the four departments, which means the second group students have selected more type-b courses than the first group, $t(585)=-4.679$, 
$p<0.001$, Cohen's $d=-0.39$ (below medium, Material), $t(884)=-5.974, p<0.001$, Cohen's $d=-0.40$ (below medium, Aeronautic), $t(567)=-2.813, p<0.01$, Cohen's $d=-0.24$ (small to medium, Instrumentation) and $t(252)=-2.202, p<0.05$, Cohen's $d=-0.28$ (small to medium, Physics).

However, the CS rate of the practical courses that are weakly related to the major (type c), and literature \& art courses (type d) have dropped significantly for three departments after the revision of the academic program. On the other hand, the selection rate of general courses such as literature and art (type d) has not changed significantly in the Department of Materials. For type-c courses, $t(586)=5.014$, $p<0.001$, Cohen's $d=0.41$ (medium, Material); $t(971)=5.047, p<0.001$, Cohen's $d=0.32$ (below medium, Aeronautic); $t(624)=3.33, p<0.001$, Cohen's $d=0.27$ (small, Instrumentation); $p=0.194$ (Physics). For type-d courses, $t(1023)=8.425, p<0.001$, Cohen's $d=0.53$ (medium, Aeronautic); $t(624)=7.33, p<0.001$, Cohen's $d=0.59$ (medium, Instrumentation); $t(262.3)=3.311, p<0.001$, Cohen's $d=0.41$ (below medium, Physics); $p=0.911$ (Material).

Moreover, our analysis revealed differing changes in the CS rate of type-a courses that are directly related to the major in the four departments. Specifically, the CS rate of Department of Aeronautics and Department of Instrumentation has increased significantly, $t(740)=-9.672, p<0.001$, Cohen's $d=-0.71$ (below large, Aeronautic), $t(350.8)=-10.81, p<0.001$, Cohen's $d=-1.15$ (large, Instrumentation), and that for Department of Materials has dropped, $t(600)=3.074, p<0.01$, Cohen's $d=0.25$ (small), while that for Department of Physics has not changed significantly $(p=0.409)$.

We perceive that students from colleges of science and engineering, influenced by the characteristics of their major, would prefer the courses of natural science and engineering technology (type-b courses) that are strongly related to the major. As the new academic program based on credit system has greatly improved students' CS autonomy, students could select courses based on their own preferences.

\subsection{The effect of course type on CS rate}

The difference of selection rate concerning the four types of courses was studied in the succeeding research when the academic program was fixed.

\subsubsection{Before the academic program was revised (Group 1)}

The one-way ANOVAs results showed significant differences of CS rate among different-type courses in the four departments before the academic program was revised (Group 1), and the course type accounted for more than $80 \%$ of the outcome variance, $\eta_{\mathrm{p}^{2}}=0.807$ (Material), $\eta_{\mathrm{p}}{ }^{2}=0.871$ (Aeronautic), $\eta_{\mathrm{p}}{ }^{2}=0.911$ (Instrumentation) and $\eta_{\mathrm{p}}{ }^{2}=0.875$ (Physics), $p<0.001$. Paired-samples t-test was then employed to compare the selection rate of the four types of courses in pairs for each department. The results reveal that the Department of Materials and Department of Instrumentations share significant differences in selection rate regarding the four types of courses $(p<0.001)$. Similarly in the Department of Aeronautics and Department of Physics, the CS rate of four types of courses exhibit significant differences from each other $(p<0.001)$ except that between type-b and type-d courses ( $p=0.075$, Aeronautic; $p=0.047$, Physics). In general, the selection rates of type-b and type-d courses are significantly higher than those of type-c and type-a courses, and the selection rate of type-c courses is significantly higher than that of type-a courses. It can thus be observed that the selection rates of the four types of courses differ substantially, ranking (from high to low) general courses such as literature and art (type-d), practical courses that are strongly related to the major (type-b), practical courses that are weakly related to the major (type-c), and the courses directly related to the major ( type-a).

\subsubsection{After the academic program was revised (Group 2)}

Similar statistical analysis was carried out on the selection rates of the four types of courses after the revision of the academic program. It was found that there are still significant differences in the selection 
rates of different types of courses, $\eta_{\mathrm{p}}{ }^{2}=0.860$ (Material), $\eta_{\mathrm{p}}{ }^{2}=0.635$ (Aeronautic), $\eta_{\mathrm{p}^{2}}=0.436$ (Instrumentation), $\eta_{\mathrm{p}}{ }^{2}=0.846$ (Physics), $p<0.001$. However, the unified change mode of each department disappears, revealed by paired-samples t-test results. The selection rates of the four types of courses in the Department of Instruments almost remain unchanged before and after the academic program revision, that is, general courses (type d) share the highest selection rate, followed by type-b courses. Type-a and c courses share similar selection rates (for the difference of CS rate between type a and c, $p=0.927$; for the rest pairs, $p<0.001$ ). For the Department of Aeronautics, with the increase of the selection rate of practical courses (type b) and the decrease of the selection rate of general courses (type d), the selection rate of type-b courses is significantly higher than that of type-d courses, while the selection rates of type-a and c courses are close and count the least (for the difference of CS rate between type a and c, $p=0.036$; for the rest pairs, $\mathrm{p}<0.001$ ). The selection rates of different types of courses in the other two departments show similar characteristics, but not quite the same. For the Department of Materials, type-b and $d$ courses still enjoy the highest selection rate, but with the significant increase of that of type-b courses, the two become close in selection rate (for the difference of CS rate between type $\mathrm{b}$ and $\mathrm{d}$, $p=0.312$; for the rest pairs, $p<0.001$ ). For the Department of Physics, type-b courses have always won the first place of the selection rate. With the decrease of the selection rate of type-d courses, the difference between type-c and d courses in selection rate decreases, while type-a courses still share the lowest selection rate (for the difference of CS rate between type $\mathrm{c}$ and $\mathrm{d}, p=0.018$; for the rest pairs, $p<0.001$ ). In general, after the revision of the academic program, type-b and $d$ courses still have the highest selection rate, followed by type-c and a courses.

Although under the condition of different academic programs, students' preferences for the four types of courses vary slightly, so it is not difficult to summarize science and engineering students' CS preferences: natural science and engineering technology courses (type b) that are strongly related to the major and general courses such as literature and art (type d) are their favorite courses, followed by the practical courses (type c) that are weakly related to their majors, and the type-a courses that are directly related to the major are the least selected. This pattern of students' CS preferences provides favorable guidance for the allocation of curriculum resources in the future.

\section{Discussions and prospects}

From the perspective of teaching management, a CS model was constructed based on the CS data in an university, and the corresponding statistical and comparative analysis were conducted by means of big data method in this study. The results verified the hypothesis that students' CS behavior (selection rate) in this model is affected by course resource allocation (course type) and external factors (before and after the revision of academic program), revealing the pattern of students' CS.

5.1 The influence of course type and academic program revision on CS reflects the pattern of science and engineering students' CS preferences

Before and after the revision of academic program, the selection rates of various courses showed significant changes, and when a specific academic program was adopted, the selection rates of different types of courses also differed substantially. It can be concluded from the data analysis results that the type of course may affect students' CS. Specifically, science and engineering students prefer STEM courses such as engineering and natural science courses the most, followed by literature and art courses to enrich academic life, study different cultures, and balance learning pressures, and career planningrelated practical courses such as law, language and management to improve students' employment skills and expand their career directions. Courses directly related to the major are the least considered ones by students as they may be unwilling to "waste" their limited CS autonomy on courses similar as required 
professional ones. Departments could allocate curriculum resources based on students' CS preferences and needs.

\subsection{Students' CS preferences entail the influence of social factors}

By analysis of the influence of external factors (academic program revision) on CS, it can be seen that the significant difference between the new and old academic programs in this study lies in the magnitude of CS freedom. The new academic program featuring credit system has greatly improved students' CS autonomy that students can choose any course offered by any department in principle, releasing students' demands for CS. Therefore, analysis of the second group of students' (new version of the academic program) CS preferences is beneficial to revealing students' real CS needs and social needs, which would be beneficial for the optimization of allocating curriculum resources and improving the efficiency of curriculum education.

In general, upon implementation of the new academic program, students' selection rates of type-b courses (natural science and engineering courses that are strongly related to the major) have increased drastically, while those of type-d (general courses such as literature and art) and type-c courses (practical courses that are weakly related to the major such as law, language and economy) have decreased. In addition to the influence of science and engineering students' specialty characteristics, based on economic and social development needs, students' preferences for type-b courses have also originated from the rise of global top-tier science and technology industries (such as the Internet and its affiliated chain industries, artificial intelligence and new energy), which have created many high-value jobs and thus stimulated students' strong learning interests. From the perspective of knowledge acquisition characteristics, the support of other natural science and engineering disciplines (type-b courses) is needed for in-depth learning of a field due to subject integration, forcing students to seize all resources to learn such courses. Besides, the dramatic changes in the way of knowledge acquisition (such as diversified learning means and flexibility of learning time and place) make online fragmented learning of employment related courses (such as law, language and management) possible, leading to the decrease of selection rates of type-c and d courses.

Therefore, based on the above discussions, to meet the needs of students' CS and economic and social development, the curriculum resources in colleges of science and engineering should focus on natural science and engineering technology courses that are strongly related to student major and literature \& art courses, followed by law, language, management and other employment related courses. The courses with contents similar to professional courses should be reduced.

\subsection{Inspirations for allocation of curriculum resources}

To optimize the allocation of curriculum resources, colleges' research on and assessment of national strategies and social development needs should be combined with analysis of students' CS data. Colleges should design the allocation of curriculum resources from the top level, establish "complete" data of teaching management, and analyze the data of students' CS by forming a scientific data model, to optimize the allocation of curriculum resources according to the derived economic and social development needs. Teaching methods and management modes should also be innovated based on the changes brought about by information technology, providing personalized, diversified and humanized education services to improve the quality of education.

However, this study is limited as merely two factors (course type and academic program revision) were selected as variables for research. Therefore, more influencing factors for CS would be introduced in the future research for statistical analysis and confidence study, such as national strategies and economic and social development needs. 
Acknowledgments: The author would like to acknowledge the support from the visiting scholar program offered by Beihang University.

Conflicts of Interest: The authors declare no conflict of interest.

\section{References:}

Albion, M. J. \& Fogarty, G. J. (2002). Factors Influencing Career Decision Making in Adolescents and Adults. Journal of Career Assessment, 10(1), 91-126.

Babad, E., Darley, J.M. \& Kaplowitz, H. (1999). Developmental aspects in students'CS. Journal of Educational Psychology, 91(1), 157-168. http://dx.doi.org/10.1037/0022-0663.91.1.157.

Babad, E. (2001). Students' CS: Differential considerations for first and last course. Research in Higher Education, 42(4), 469-492. http://dx.doi.org/10.1023/A: 1011058926613.

Babad, E., \& Tayeb, A. (2003). Experimental analysis of student's CS. British Journal of Educational Psychology, 73, 373-393.http://dx.doi.org/10.1348/000709903322275894.

Calvo, G. \& Frenkel, J. (1991). From Centrally Planned to Market Economy: The Road from CPE to PCPE. IMF Econ Rev, 38, 268-299.

ISCED. (1997). https://wenku.baidu.com/view/5c8028e9856a561252d36fa2.html.

Kelle, U. (2006). Combining qualitative and quantitative methods in research practice: purposes and advantages. Qualitative research in psychology, 3 (4), 293-311.

KERIN, R., HARVEY, M. \& Fredric, C. N. (1975). Student CS in a non-requirement program: An exploratory study. The Journal of Educational Research, 68 (5), 175-177.

Lent, R. W., Brown, S. D. \& Hackett, G. (2000). Contextual supports and barriers to career choice: A social cognitive analysis. Journal of Counseling Psychology, 47(1), 36-49.

Marginson, S. (2013). The impossibility of capitalist markets in higher education. Journal of Education Policy, 28(3), 353-370. http://dx.doi.org/10.1080/02680939.2012.747109.

McGoldrick, K. \& Schuhmann, P.W. (2002). Instructor gender and student registration: an analysis of preferences. Education Economics, 10 (3), 241-260.

Pass, M. W., Mehta, S.S. \& Mehta, G.B. (2012). CS: student preferences for instructor practices. Academy of Educational Leadership Journal, 16(1), 31-38. 